\title{
Structural Design and Performance Analysis of a Particle Micro-thruster
}

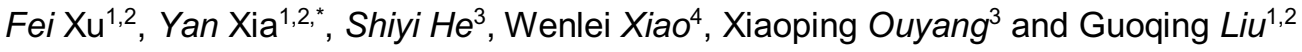 \\ ${ }^{1}$ Beijing Institute of Spacecraft Environment Engineering, 100094 Beijing, China \\ ${ }^{2}$ Science and Technology on Reliability and Environmental Engineering Laboratory, 100094 Beijing, China \\ ${ }^{3}$ Northwest Institute of Nuclear Technology, 710024 Xi'an, China \\ ${ }^{4}$ Beihang University, 100083 Beijing, China
}

\begin{abstract}
The $\alpha$ particle propelling is a technology under developing by the author's research group, which uses $\alpha$ decay isotopes emitting high-speed particles to generate thrust. In this paper we mainly designed the thrust magnitude control and vector control structure suitable for this propelling principle and preliminarily analyzed its control performance. The proposed structure mounts the trust film composed of the decay nuclides onto a quasi-spherical frame surface that is conducive to vector synthesis. The traveling wave type ultrasonic motor drives the diaphragm to control the thrust magnitude of each thrust unit independently and efficiently. The vector control of the joint thrust is implemented by taking advantage of the diversity of combination of multiple thrust units. Theoretical calculation and analysis showed that the thruster structure could achieve the thrust magnitude control accuracy of $0.05 \mu \mathrm{N}$, and the thrust angle control accuracy of $0.5-1.5$ degrees.
\end{abstract}

\section{Introduction}

The principle of $\alpha$ particle propelling is to coat radioactive material onto a thin film exposed on the surface of spacecraft, and use its $\alpha$ decay to emit high-energy particles into space to form a recoil force. The thruster based on a decay principle has the characteristics of high specific impulse, long life, low power requirement and accuracy control potential. It has unique application value compared to solar sail, electric propulsion and other micro-propulsion technologies. This concept was proposed by European scholars in the last century[1], but it has not been further developed. In recent years, the author's research group has been devoted to developing this principle into a viable micro-thruster device.

At present, high accuracy micro-thruster is the key technology of drag-free flight spacecraft, and drag-free flight technology has been widely used in basic physical verification[2], gravity field measurement missions[3,4]. Until present, the main drag-free hired propelling methods have been electric propulsion[5,6] and cold gas propulsion[7,8], both adopting flow control to achieve control of the thrust. The principle of $\alpha$ particle propelling is different, so it is impossible to refer to flow control mode. Alpha decay process is spontaneous, and one cannot restrain the number of $\alpha$ particles emitted, but $\alpha$ particles have weak penetration, thus one can restrain the number of $\alpha$ particles escaping from the spacecraft system, by simply applying thin layer to cover the decay film. Therefore, $\alpha$ particles propelling is suitable to employ area control method.

Vector propulsion is another key technology of space propulsion[9,10]. Film-type $\alpha$ particle thruster, which does not consume a lot of power, is very suitable for pico\&nano, mobile phone and chip satellites. Pico\&nano satellites cannot afford the power consumption of electric propulsion. According to statistics, only $9 \%$ of the nano-satellites has orbit-control, so the on-orbit life is generally short. The application of $\alpha$ decay thruster can extend the life of pico\&nano satellite in orbit from one year to ten years, thus reducing the need for repeated launch and network replenishment of the same functional satellites, and further greatly reducing the application cost of the small satellite. Due to the limited angle

* Corresponding author: xiayan_cast@ 163. com 
control capability of the pico\&nano satellite platform, their micro-thruster for orbit control should have the directional adjustability in a certain range, i.e., the vector control.

Considering the physical characteristics of $\alpha$ decay, the control structure of $\alpha$ particle micro-thruster is designed to meet the requirements of drag-free flight and orbit control of pico\&nano satellite, and the preliminary performance analysis was carried out.

\section{Thrust Film of $\alpha$ Decay}

The main characteristic of $\alpha$ particle is its weak penetration. Taking $5.3 \mathrm{MeV} \alpha$ particles of ${ }^{210} \mathrm{Po}$ decay as an example, their range of penetration through Polonium metal itself is $23.4 \mu \mathrm{m}$. Alpha particles generated at the depth beyond the range have almost no probability to escape the film into the space, so generate no thrust. The thrust film is only efficient with surface thickness less than the range, and the metal base is used to block $\alpha$ particles emitted towards the other half space. The thrust film structure is shown in Fig. 1. After a theoretical calculation and simulation study, and considering thrust, cost and life, our team preferred ${ }^{210} \mathrm{Po}$ with $10 \mu \mathrm{m}$ thickness as thrust film to build $\alpha$-particle micro-thruster with. The thrust of the thin film is $0.03 \mu \mathrm{N} / \mathrm{cm}^{2}$ along the $-\mathrm{X}$ direction. The specific theoretical derivation and testing process can refer to the relevant papers of our research group.

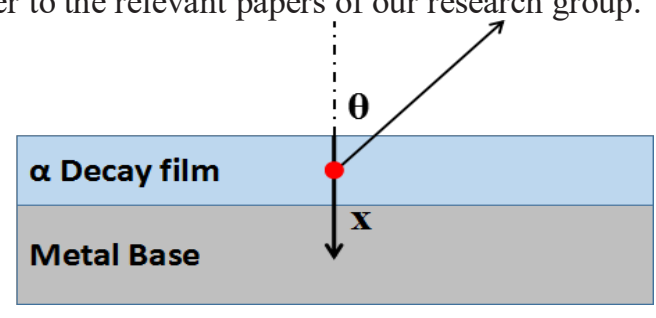

Fig. 1. Thrust film of $\alpha$ decay

\section{The Structure Design for Thrust Control}

\subsection{Basic structure for thrust control}

The thrust of $\alpha$ particle micro-propulsion should take area control mode. We use the structure of diaphragm driven by ultrasonic motor to achieve this function. As shown in Fig. 2, the diaphragm and the ultrasonic motor are circular laminated structures, both of which are positioned directly above the thrust film, while the diaphragm is positioned under the upper ultrasonic motor. The diaphragm can open and close the central passage, which is driven by traveling wave rotary ultrasonic motor. The mechanical system can control the thrust of $\alpha$ particles by controlling the exposure area of the thrust film.

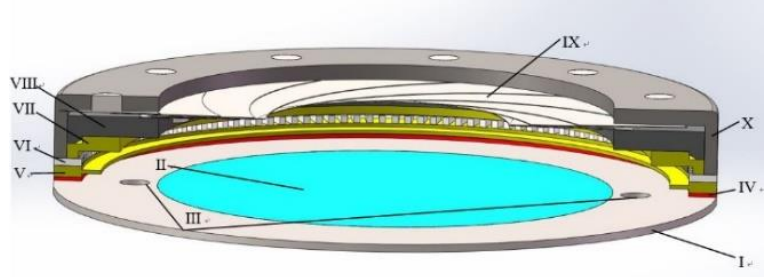

Fig. 2. Section diagram

I: Base; II: Thrust film of $\alpha$ decay; III: Two-phase alternating input wire holes; IV: Piezoelectric ceramic; V: Ultrasonic motor stator; VI: Friction tooth; VII: Ultrasound motor Actuator; VIII: Diaphragm movable ring; IX: Diaphragm blade; X: Diaphragm fixing ring

Ultrasonic motor drives diaphragms in no need of additional deceleration and it is compact in structure. The diaphragm structure is simple and occupies small space. This is why the $\alpha$ particles micro-thruster chooses ultrasonic motor and diaphragm combination. Ultrasound motor can work at $-150^{\circ} \mathrm{C}$ and $10^{-6} \mathrm{~Pa}$ [11], and is not susceptible to electromagnetic interference, thus applicable in space environment[12]. The ultrasonic motor has fast response (millisecond level), drives directly without gear deceleration, and can accurately stop and locate. In theory, the aperture size of the diaphragm can be continuously adjustable, and the corresponding thrust can be arbitrarily controllable. Each basic control structure consists of 11 diaphragms driven together by one ultrasonic motor. Fig. 3 shows the minimum and maximum thrust states of the basic control unit. The diaphragm blades of this system are made of 304 stainless steel, and the motor material is PZT lead zirconate titanate.
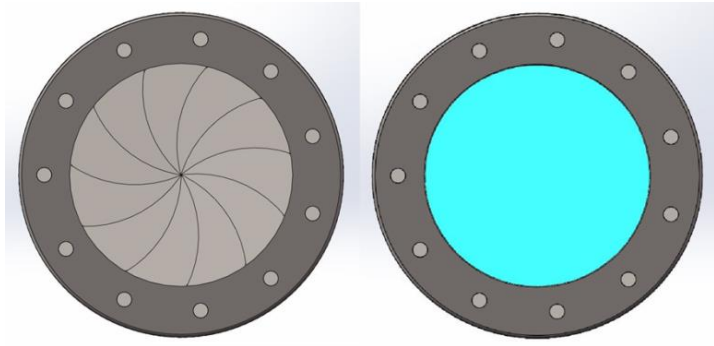

Fig. 3. Minimum and maximum propulsion status of the basic control mechanics

\subsection{Quasi-spherical vector micro-thruster}

The quasi-spherical structure high precision vector thruster includes quasi-spherical frame, thrust film of $\alpha$ decay, basic structure for thrust control, heat transfer 
unit and thermoelectric conversion center.

\subsubsection{Quasi-spherical frame}

The frame is a convex structural configuration. It is of heat resistant, corrosion resistant and high strength titanium alloy. Its outer surface consists of six identical regular pentagons, five identical regular hexagonal planes to mount the thrust film on and five identical trapezoidal non-functional surfaces to complement the structure, forming a quasi-spherical configuration. On top of each tangential circular area of the functional pentagons and hexagons, thrust film and its corresponding basic control unit are located and attached. The thrust film is placed concentric to the aperture opened by the diaphragm blade and the diameter of the film and the maximum aperture is identical. The inner surface of the frame is connected with the heat transfer unit, and the heat is exported to the thermoelectric conversion center. As a design example, the pentagons have a side length of $5.04 \mathrm{~cm}$, with inner tangential circle radius of $3.47 \mathrm{~cm}$ and the aperture radius of $3.00 \mathrm{~cm}$; the hexagons have side length of $5.04 \mathrm{~cm}$, with inner tangential circle radius of $4.37 \mathrm{~cm}$ and the aperture radius of $2.36 \mathrm{~cm}$. The ring width of the diaphragm is $1.10 \mathrm{~cm}$ in pentagon and $1.37 \mathrm{~mm}$ in hexagon. The azimuth angle of dome pentagon is equal to $0^{\circ}$, the azimuth angle of 5 peripheral hexagons is $37.38^{\circ}$, and the azimuth angle of 5 more peripheral pentagons equals to $63.43^{\circ}$. The inner radius of the frame is $11.10 \mathrm{~cm}$, the outer radius of the thermoelectric conversion center is $5.85 \mathrm{~cm}$, and the thickness of the alloy frame is $3.5 \mathrm{~mm}$ (the thinnest part), as shown in Fig. 4.

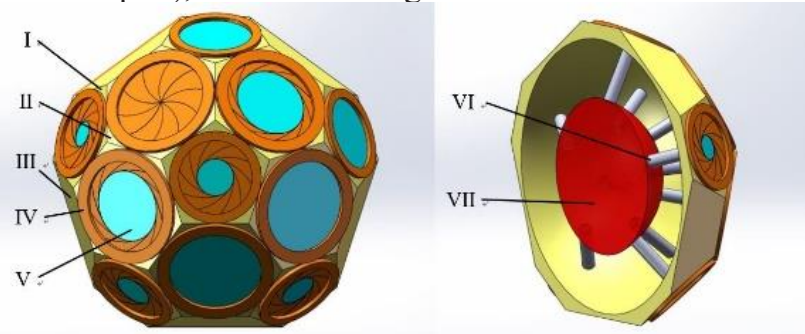

Fig. 4. Front and back of quasi-spherical vector micro-thruster

I: Pentagon; II: Hexagon; III: Non-functional surface; IV: Basic structure of thrust control; V: Propulsion film of $\alpha$ decay; VI: Panel heat transfer unit; VII: Thermoelectric conversion center;

\subsubsection{The heat transfer unit and thermoelectric conversion center}

The thruster concept further transfers the residual heat of decay into electricity through thermoelectric conversion center and heat transfer unit. Alpha decay films produce both power and heat, the thermoelectric conversion center has a hot end/hot layer, and the residual heat of film passes through the 11 heat transfer units to the hot end of 1 thermoelectric conversion center and finally the thruster reaches a balanced thermal distribution. The thermoelectric conversion center has a cold end/cold layer, which radiates heat to the space through heat rejection panels. A few thermocouple chips are installed between hot and cold layers to generate electricity driven by temperature difference. The cold end heat is still high and can further to be used as satellite heat source when in need.

The heat transfer unit shall have good structural and thermal conductivity characteristics. It is hollow in center and connects the frame and thermoelectric conversion center. It provides good structural stability for the quasi-spherical structure, conduct heat, and allows the power supply and control wires to pass through its hollow channel to connect the motor servo drivers.

In the above mentioned design example, the heat transfer unit is a hollow copper tube with an outer radius of $6 \mathrm{~mm}$ and an inner radius of $5 \mathrm{~mm}$. The thermoelectric conversion center is a multi-layer spherical shell structure, which consists of a hot end, a thermoelectric converter layer and a cold end. Its material is copper and its thickness is $2 \mathrm{~mm}$. The equilibrium temperature without any heat rejection is 653K (The cold end shall be designed with heat sinks, heat pipes and other thermal control structures to reject heat to make this temperature durable for material and its supported spacecraft). The temperature difference between the cold end and the hot end is $150 \mathrm{~K}$. Thermoelectric converter between hot end and cold end is planned as TGPR chip made by TEG-Pro company. Each is $56 \mathrm{~mm} \times 56 \mathrm{~mm} \times 5 \mathrm{~mm}$. Five chips are arranged in a sandwich structure between the cold end and hot end, as shown in Fig. 5, each generating $5.6 \mathrm{~W} /$ piece, in total $28 \mathrm{~W}$.

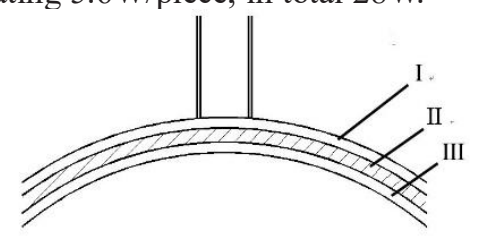

Fig. 5. Schematic diagram of the thermoelectric conversion center structure

I: The hot end; II: Thermoelectric conversion device; III: The cold end

\section{Control performance analysis}


In order to simplify the calculation, only $2^{11}=2048$ kind of joint thrust is considered, allowing only' 0 ' and ' 1 ' status, i.e., fully open and closed status, of each basic thrust unit. This preliminary analysis does not consider the continuous control capabilities of each unit thrust, but in fact the move of diaphragm driven by ultrasonic motor is continuous, i.e., there are in theory infinite kinds of combination status, the proposed thruster shall have higher thrust control accuracy and angle control accuracy than the calculation in the following.

The analysis was modelled and executed with Matlab ${ }^{\odot}$. It decomposed the thrust of 11 basic units into 3 components along $x, y, z$ axis. Then all possible joint thrust is calculated, by traversing all combinatorial status of 11 basic units and adding its corresponding basic thrust combination. Finally, statistical was done based on the joint thrust set of 2048 possibilities to analyze the performance in term of thrust control and vector control accuracy. Fig. 6 (a-d) shows the histogram of the joint thrust, the thrust along the spherical normal direction, the azimuth angle, and the zenith angle of thrust. Within the range that all bins having none-zero combination amount, we claim its accuracy is better than half the bin size, and within the range that some bins has zero combination amount, we claim the accuracy is better than half of the gap (zero bin width).

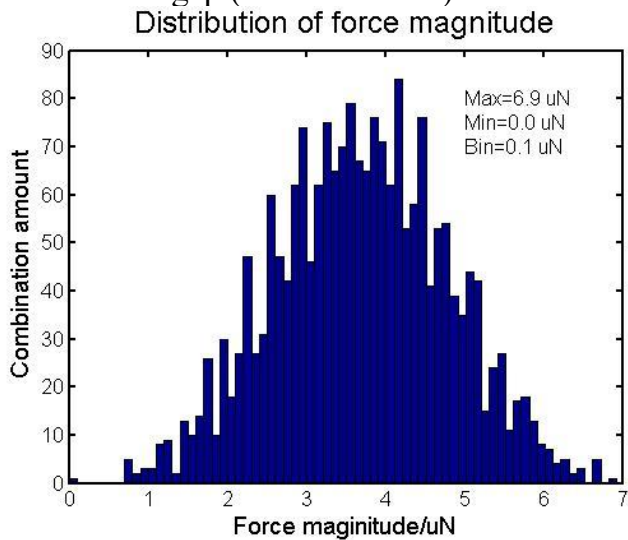

(a) Thrust distribution

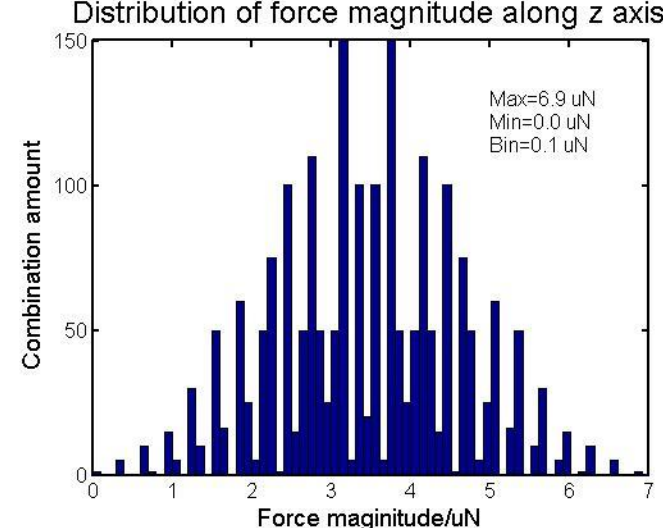

(b) Normal component thrust distribution Distribution of $\phi$

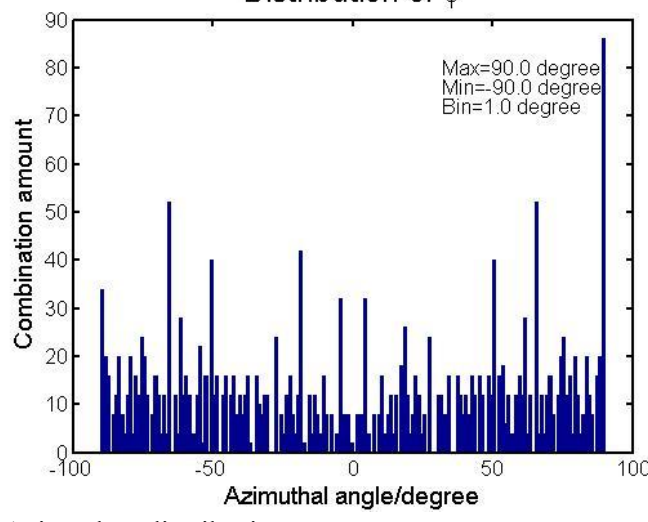

(c) Azimuth $\varphi$ distribution

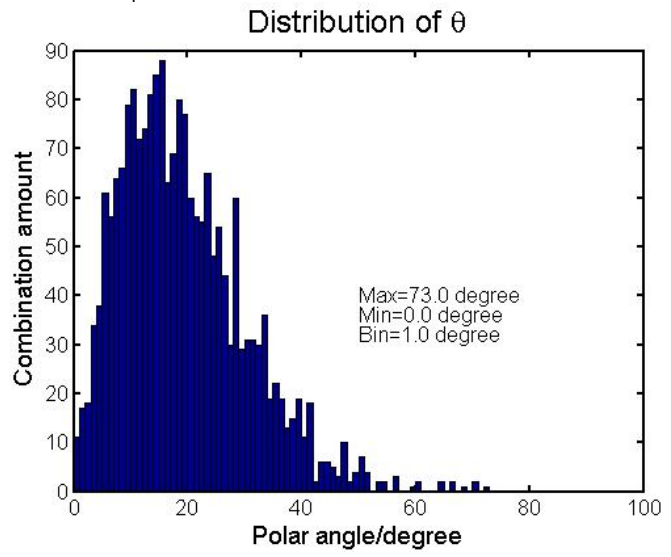

(d) Zenith angle $\theta$ distribution

Fig. 6. Histogram analysis of thrust vector

The joint thrust is within the range of $0-6.9 \mu \mathrm{N}$, in the sub range of $0.7-6.5 \mu \mathrm{N}$ the thrust is continuously adjustable. Continuously adjustable means that when the system specifies a magnitude of force, it can be realized by combining " 0 " and " 1 " status of 11 basic units, and the realization thrust accuracy is better than $0.05 \mu \mathrm{N}$ (half the bin size).

The thrust magnitude along the $\mathrm{z}$ axis is within 
the range of $0-6.9 \mu \mathrm{N}$, in the sub range of $1.8-5.1 \mu \mathrm{N}$ the $z$-trust is continuously adjustable with control accuracy better than $0.05 \mu \mathrm{N}$, and in the range of $0-1.81 \mu \mathrm{N}$ and $5.1-6.9 \mu \mathrm{N}$, the corresponding combinatorial number is few, but the control accuracy of this range maintain better than $0.15 \mu \mathrm{N}$.

According to distribution in the phi angle, the azimuth angle is distributed within -90-90 degrees. The distribution is not uniform, because the system is quasi- not perfect spherical, to part of the angle corresponding combination are more than to others. The largest opening in the histogram is 3 degrees, so the azimuth vector control accuracy is claimed to be 1.5 degrees.

From the distribution of theta angle, it can be seen that the polar angle of the thrust is distributed in range of 0-73 degrees. Over $99 \%$ is concentrated in the smaller angle range of 0-53 degrees, and the control accuracy within this range is better than 0.5 degree. The corresponding combinatorial number within the range of 54-73 degrees is few, with the control accuracy is better than 2 degrees.

\section{Conclusion}

Compared with micro-propulsion as electric and solar sail propelling, $\alpha$ particle propelling technology has unique advantages in large specific impulse, long life, and low power consumption. If the related engineering application technologies are proper addressed, the technology is promising in space use and become a powerful supplement in the field of micro-propulsion.

The first engineering challenge should be considered is the control structure, mode and accuracy. In this paper, the basic control and vector control structure of $\alpha$-particle thruster were designed. The idea is to attach the thrust film of decay nuclides with individual area control motors onto quasi-spherical frame to benefit vector synthesis of units' thrust, the diversity of combination is the basis of high accuracy of control. The traveling-wave ultrasonic motor driving diaphragm is used to control the exposure area of the thrust film independently and efficiently. The theoretical analysis was carried out showing the thruster structure reaches the thrust control accuracy of $<0.05 \mu \mathrm{N}$ in large portion of thrust range. The angle control accuracy is 0.5 degree in polar and 1.5 degree in azimuth.

The $\alpha$-particle micro-thruster is still in the conceptual stage. More detailed control structure design, its feasibility and performance analysis should be carried out. Moreover the heat dissipation and charge neutralization associated with $\alpha$-decay process should be properly solved. The generation of power using decay waste heat (especially in the application of pico\&nano satellite) is also a possible additional performance worthy of further discussion. These are the future research directions of our research group.

\section{References}

1. C. Bruno, M. Rosa-Clot, ACTA ASTRONAUT, 38, 231-234(1996)

2. D.B. DeBra, ADV SPACE RES, 32, 1221-1226 (2003)

3. L. Massotti, A. Molano, E. Canuto, IEEE T CONTR SYST T, 18, 501-509(2010)

4. H. Liu, P.B. Chen, Q.Q. Sun, et al, ACTA ASTRONAUT, 126, 35-39(2016)

5. C. Scharlemann, N. Buldrini, R. Killinger, et al, ACTA ASTRONAUT, 69,822-832(2011)

6. K. Cui, H. Liu, W.J. Jiang, et al, ACTA ASTRONAUT,144,193-200(2018)

7. R. Ranjan, S.K. Chou, F. Riaz, et al, Energy Procedia, 143,754-761 (2017)

8. R. Ranjan, K. Karthikeyan, F. Riaz, et al, APPL ENERG, 220, 921-933(2018)

9. A.K. Vinayagam, N.K. Sinha, P I MECH ENG G-J AER, 228, 960-979(2014)

10. L. Chen, H. Zhang, D.P. Duan, P I MECH ENG G-J AER, 228, 2045-2054(2014)

11. S.S. Lih, B.C. Yoseph, W. Grandia, SPIE, 3041, 912-917 (2004)

12. C.S. Zhao, Ultrasonic Motors Technologies and Applications (Science Press, Beijing, 2007) 